\title{
Polypoid nodule scar after gastric endoscopic submucosal dissection: results from a multicenter study
}

\section{(ㄷ)(1) $\odot$}

\section{Authors}

Vitor Arantes ${ }^{1}$, Noriya Uedo², Yoshinori Morita ${ }^{3}$, Takashi Toyonaga4, Yoshiko Nakano ${ }^{3}$, Moises Salgado Pedrosa ${ }^{5}$, Ichiro Oda ${ }^{6}$, Yutaka Saito ${ }^{6}$, Haruhisa Suzuki ${ }^{6}$, Katsumi Yamamoto ${ }^{7}$, Yu Sato ${ }^{7}$, Peter V. Draganov ${ }^{8}$

Institutions

1 Endoscopy Unit, Alfa Institute of Gastroenterology, School of Medicine, Federal University of Minas Gerais, Hospital Mater Dei Contorno, Belo Horizonte, Brazil

2 Department of Gastrointestinal Oncology, Osaka Medical Center for Cancer and Cardiovascular Diseases, Osaka, Japan

3 Department of Gastroenterology, Kobe University School of Medicine, Kobe, Japan

4 Department of Endoscopy, Kobe University School of Medicine, Kobe, Japan

5 Pathology Department, School of Medicine, Federal University of Minas Gerais, Laboratory CEAP, Belo Horizonte, Brazil

6 Endoscopy Division, National Cancer Center Hospital, Tokyo, Japan

7 Department of Gastroenterology, Japan Community Healthcare Organization Osaka Hospital. Osaka, Japan

8 University of Florida, Gainesville, Florida, United States

submitted 1.2.2018

accepted after revision 15.3 .2018

Bibliography

DOI https://doi.org/10.1055/a-0607-2452 |

Endoscopy International Open 2018; 06: E1198-E1203

(c) Georg Thieme Verlag KG Stuttgart · New York ISSN 2364-3722

\section{Corresponding author}

Vitor Arantes, MD, MSc, PhD, Endoscopy Unit, Alfa Institute of Gastroenterology, School of Medicine, Federal University of Minas Gerais, Hospital Mater Dei Contorno, Rua Florália 18, apt. 1201, Anchieta. Belo Horizonte - MG - Brazil

30.310-690

Fax: +553132804117

arantesvitor@ufmg.br

arantesvitor@hotmail.com

\section{ABSTRACT}

Background and study aims A post-endoscopic submucosal dissection (ESD) scar is expected to look homogeneous, however, some patients develop benign polypoid nodule scar (PNS). Incidence of PNS is unknown, yet these scars have direct clinical implications because they may render evaluation of post-ESD neoplastic recurrence difficult. Therefore, we reviewed the clinical experience of 5 ESD referral centers and evaluated their PNS incidence and clinical management.

Patients and methods This was a retrospective multicenter case series enrolling patients that underwent R0, curative gastric ESD from 2003 to 2015 in 5 academic centers. PNS was defined as ESD site nodularity with hyperplastic or regenerative tissue histology.

Results A total of 2275 patients underwent gastric ESD with endoscopy control and 28 patients ( 18 men/10 women) developed PNS for overall incidence of $1.2 \%$. Incidence of PNS ranged from $0.15 \%$ to $11.4 \%$ between centers. All patients that developed PNS had primary neoplastic lesions located in the distal stomach. Considering only lesions situated in the antrum $(n=912)$, incidence of PNS was $3.1 \%$. After mean follow-up of 43 months (range 6-192), no malignant recurrence in the PNS has been identified. In five patients (17.8\%) PNS disappeared after a mean of 18 months. Conclusion PNS occurs exclusively after ESD in the distal stomach in approximately $3.1 \%$ of patients. Although PNS appearance can be concerning, no malignant recurrence was observed after curative R0 resection. Therefore, PNS should be viewed as a benign alteration that does not require any type of intervention, other than endoscopic surveillance. 


\section{Introduction}

Endoscopic submucosal dissection (ESD) is the treatment of choice for patients with superficial gastric neoplasms with no or low risk of lymph nodes metastasis $[1,2]$. It permits en bloc resection of lesions regardless of their size, thus allowing accurate and reliable histological assessment of resection margins and final staging to determine the curability of the endoscopic resection. Endoscopic surveillance is recommended after gastric ESD to inspect the healing process, rule out residual or recurrent tumor and screen for metachronous lesions.

After a curative ESD, the postoperative scar typically appears homogeneous and whitish, often with converging folds. Anecdotally we have noted at post-ESD endoscopic examination that some patients develop irregular scars, with nodules some of which can be large and protruding. Based on our observation, we named this entity polypoid nodule scar (PNS) [3, 4]. In our limited initial experience, biopsy specimens taken from these scars have demonstrated regenerative mucosa with hyperplastic changes without recurrent neoplastic cells. Presence of PNS creates a clinical dilemma because it may obscure neoplastic recurrence, particularly considering that many of PNS-related nodules can be quite large and protruding. Furthermore, in our initial series we noted a significant variation in incidence of PNS from $1.7 \%$ in the Japanese center up to $13.3 \%$ in the Brazilian center [4]. Therefore, we conducted a multicenter study to further investigate on a larger scale incidence and clinical implications of PNS after curative gastric ESD.

\section{Patients and methods}

This was a multicenter retrospective case series involving five high-volume ESD centers ( 1 in Brazil and 4 in Japan) as summarized in $>$ Table 1. The study was carried out in accordance with the Helsinki Declaration, and institutional review board (IRB) approvals were obtained in each of the participating institutions. All patients provided procedure informed consent. Informed consent for study participation was waived because of the retrospective study design. Clinical information from 2003 to 2015 was extracted retrospectively from the endoscopy database of each institution. Data were obtained by means of endoscopic and histological reports review, and assessment of endoscopic images of the post-ESD scar.

\section{Inclusion and exclusion criteria}

Eligibility for ESD was assessed by means of white-light endoscopy, image-enhanced endoscopy (IEE), magnifying endoscopy, indigo carmine spraying and endoscopic ultrasound (in selected cases, at the discretion of the endoscopist). Patients were included in the study when all of the following were present: 1. successful en bloc ESD procedure with confirmatory histology of R0 and curative resection [1]; 2. postoperative endoscopic examination with identification of a protruded polypoid nodule scar corresponding to the site where the ESD was undertaken; and 3. biopsies of the PNS with histological assessment demonstrating hyperplastic or regenerative tissue. Before data collection, each investigator involved in the study reviewed and agreed on the typical endoscopic features of PNS previously described [4]. Experienced gastrointestinal pathologists from each center reviewed PNS biopsies. In addition, endoscopic images of PNS cases from each Institution were sent to the leading investigator (VA) aiming to check for interobserver agreement. Data were examined for patient demographics, histologic findings, Helicobacter pylori status, postoperative medical management, clinical outcome and endoscopy follow-up findings. Patients lost to follow-up that did not undergo at least one post-ESD examination were excluded from this analysis. $H$. pylori infection was assessed by a combination of urea test, histological assessment of gastric samples or serum antibody.

\section{ESD procedure}

The ESD technique has been described in detail elsewhere [5]. Briefly, markings were placed at least $2 \mathrm{~mm}$ beyond the borders of the lesion after careful endoscopic assessment by chromoendoscopy and/or magnifying endoscopy (ME). Viscous solution or $0.9 \%$ saline solution was used for submucosal (SM) injection at the discretion of the endoscopist. ESD was undertaken with specialized knives such as Flush-Knife Ball Tipped (Fujifilm Co.,

- Table 1 Endoscopic submucosal dissection procedures distribution among five participating institutions and incidence of polypoid nodule scar, according to region of stomach.

\begin{tabular}{|c|c|c|c|c|c|}
\hline ESD procedures & $\begin{array}{l}\text { Center } 1 \\
\text { Alfa Institute of } \\
\text { Gastroenterolo- } \\
\text { gy, Belo Hori- } \\
\text { zonte-Brazil }\end{array}$ & $\begin{array}{l}\text { Center } 2 \\
\text { Osaka Medical } \\
\text { Center for Cancer } \\
\text { and Cardiovascular } \\
\text { Diseases }\end{array}$ & $\begin{array}{l}\text { Center } 3 \\
\text { Kobe University } \\
\text { School of } \\
\text { Medicine }\end{array}$ & $\begin{array}{l}\text { Center } 4 \\
\text { National Can- } \\
\text { cer Center } \\
\text { Hospital, } \\
\text { Tokyo }\end{array}$ & $\begin{array}{l}\text { Center } 5 \\
\text { Japan Commu- } \\
\text { nity Healthcare } \\
\text { Organization } \\
\text { Osaka Hospital }\end{array}$ \\
\hline Total number of gastric ESD, n & 79 & 343 & 241 & 1290 & 322 \\
\hline ESD in distal stomach, $\mathrm{n}(\%)$ & $49(62.0 \%)$ & $158(46.0 \%)$ & $69(28.6 \%)$ & $495(38.4 \%)$ & $141(43.7 \%)$ \\
\hline ESD in proximal stomach, $n(\%)$ & $30(38.0 \%)$ & $185(54.0 \%)$ & $172(71.3 \%)$ & $795(61.6 \%)$ & $181(56.3 \%)$ \\
\hline Total number of PNS cases, n (\%) & $9(11.4 \%)$ & $6(1.7 \%)$ & $5(2.0 \%)$ & $2(0.15 \%)$ & $6(1.8 \%)$ \\
\hline PNS in distal stomach, n (\%) & $9(18.3 \%)$ & $6(3.8 \%)$ & $5(7.24 \%)$ & $2(0.4 \%)$ & $6(4.25 \%)$ \\
\hline
\end{tabular}


Japan) or ceramic-ball insulated tip knife (IT knife, Olympus Co., Japan), according to the preference of the endoscopist. Mucosal incision was undertaken around the tumor in a circumferential or semi-circumferential manner. SM dissection was performed in the deep submucosa with identification and hemostasis of the penetrating vessels. After complete tumor resection, the ulcer site was assessed and visible vessels were coagulated with a hemostatic forceps. The specimen was stretched and fixed in a Styrofoam plate, immersed in $10 \%$ formalin solution and sent to the pathology department.

\section{Histological assessment and definitions}

After being embedded in $10 \%$ paraffin, the specimens were cut into 2-mm slices and stained with hematoxylin and eosin. Additional immunochemistry studies with D2-40 and CD34 were carried out for lymphatic and vascular invasion assessment, at the discretion of the pathologist. Tumor size, depth of invasion, lymphatic and vascular invasion, grade of differentiation, and resection margins were histopathologically examined [6]. En bloc resection was defined as complete removal of the visible tumor including the markings into one non-fragmented piece [2]. R0 resection was defined histologically as complete tumor removal with both lateral and deep margins free of neoplastic cells. Endoscopic resection was considered curative when the pathology report demonstrated adenoma with low- or highgrade dysplasia, well or moderately differentiated adenocarcinoma, depth of invasion restricted to mucosa or superficial submucosa (SM1), with negative lateral and deep margins and no lymphovascular invasion [2, 3, 7]. ESD was considered noncurative when any one of the following criteria were present $[2,3,5]$ : undifferentiated cancer greater than $2 \mathrm{~cm}$, deep submucosal tumor invasion (SM2), positive lateral or horizontal margins, and lymphovascular invasion. Patients with non-curative resection were not included in this study. PNS was defined as a protuberant polypoid-appearing nodule situated in the post-ESD scar site, with or without converging folds and with histological assessment of forceps biopsies demonstrating only regenerative or hyperplastic tissue growth without any neoplastic tissue, confirmed by experienced gastrointestinal pathologists.

\section{Post-procedure care}

All patients were hospitalized for postoperative observation. Intravenous or oral proton pump inhibitors (PPI) were administered to all patients during the first postoperative days followed by an 8-week course of oral PPI after hospital discharge. Second-look endoscopy was performed at the discretion of the endoscopist. If the ESD procedure was considered curative, the first follow-up endoscopy was scheduled usually at 3 and 12 months, and annually thereafter. The ESD scar was inspected carefully for any abnormality such as residual tumor or polypoid nodule growth utilizing IEE or ME, and multiple forceps biopsies were performed.
- Table 2 Clinicopathological characteristics of patients with polypoid nodule scar.

Sex (male/female), $n$

$18 / 10$

Age (y), median (range)

$73(54-80)$

Tumor size (mm), median (range)

$20(08-55)$

Tumor location (distal stomach), $\mathrm{n}$ (\%)

- Anterior wall

$7(25.0 \%)$

- Posterior wall

$2(7.0 \%)$

- Greater curvature

$12(43.0 \%)$

- Lower curvature

$6(21.5 \%)$

- Incisure

$1(3.5 \%)$

Tumor histology, $\mathrm{n}(\%)$

- Well-differentiated adenocarcinoma $18(64.3 \%)$

- Moderately differentiated adenocarcinoma

$1(3.5 \%)$

- Adenoma with high-grade dysplasia $5(17.9 \%)$

- Benign gastric polyp $4(14.3 \%)$

PNS disappearance over time, $n$ (\%) $5(17.9 \%)$

H. pylori infection, $n$ (\%) $14(50 \%)$

ESD, endoscopic submucosal dissection; PNS, polypoid nodule scar; $n$, number; H. pylori, Helicobacter pylori; $y$, years; mm, millimeters

\section{Statistical analysis}

The primary outcome measure was assessment and characterization of post-ESD scars at follow-up endoscopy. Descriptive statistical analysis of the data, including percentage, median and $95 \%$ confidence interval, were calculated. Categorical data were expressed as frequencies and percentages.

\section{Results}

During the study period, 2865 patients from five centers underwent gastric ESD. A total of 590 cases were excluded from analysis due to non-curative resection or absence of postoperative follow-up. Therefore, 2275 patients that underwent curative gastric ESD and received postoperative endoscopic follow-up entered the study. Among this population, 28 patients (18 men/10 women) developed PNS for an overall occurrence of $1.2 \%$ [ $95 \%$ confidence interval: $0.8-1.8 \%$ ]. The earliest appearance of PNS was noted 3 months after ESD and the latest after 18 months (median: 6 months). The proportion of PNS varied between the participating institutions and is summarized in - Table 1.

- Table 2 demonstrates the clinicopathological characteristics of patients with PNS. Tumor size ranged from $8 \mathrm{~mm}$ to $55 \mathrm{~mm}$ (median $20 \mathrm{~mm}$ ). Postoperative medical treatment included PPI for all patients during 8 weeks (either standard or double dose at the discretion of the endoscopist) and hospital admission for a minimum of 48 hours. Moreover, Center \#1 administered sucralfate postoperatively for 2 weeks and Center 

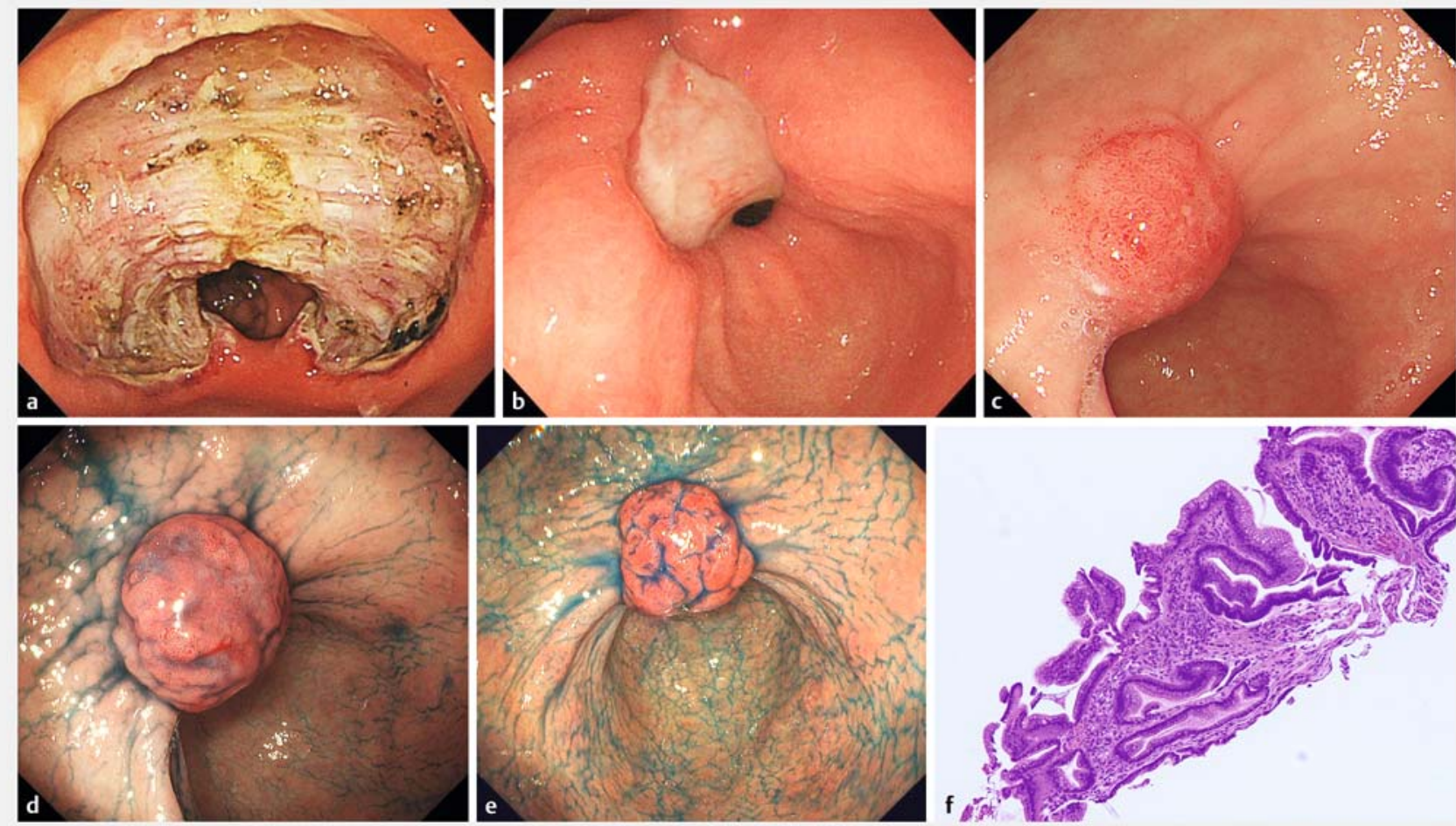

- Fig. 1 Illustrative case of polypoid nodule scar (PNS) after endoscopic submucosal dissection (ESD) from Kobe University Hospital, Kobe, Japan. a Endoscopic view of defect after enbloc resection of a lesion situated in the pre-pyloric area. b Ulcerated scar 1 month after ESD. c Small nodule noted at ESD scar 1 year after ESD. d Protuberant nodule (PNS) observed 2 years after ESD. e PNS remains unchanged 3 years after ESD. f Histology from nodule biopsy demonstrates hyperplastic polypoid neoformation, showing elongation, tortuosity and dilation of the gastric foveolae, and stroma with fibrosis and inflammatory cells.

\#3 added sucralfate and sodium alginate for 1 week and rebapimide for 8 weeks. A total of 14 of 28 PNS patients (50\%) tested positive for $H$. pylori. All patients that developed PNS had primary neoplastic lesions located in the distal stomach (27 antrum/1 incisure). PNS was not observed in any patient with tumors located in the body, fundus or cardia. Considering only lesions situated in the distal stomach, the proportion of PNS cases was $3.1 \%$ [95\% confidence interval: $2.0-4.4 \%$ ] (28 out of 912$)$.

To assess the possibility of learning curve effect on occurrence of PNS, we divided the study period into two phases (2003-2010 and 2011-2016). In 6 of 28 patients (21.4\%), ESD was undertaken in the early period of experience from the operators (2003 to 2010). The great majority of cases occurred after 2011, when all operators had already overcome the learning curve for gastric ESD (first 40 cases).

All participating patients have been followed periodically with endoscopy and no neoplastic recurrence was identified in any of the PNS patients (follow-up range: 3 to 192 months, median: 41 months). In five cases (17.9\%) PNS disappeared over time (range: 10-30 months, median: 24 months). In the remaining 23 cases, PNS remained present at follow-up endoscopic examination without significant variations in size or shape, although occasionally some decrease in size was noted. $\triangleright$ Fig. 1a, > Fig. 1b, > Fig.1c, > Fig.1d, > Fig. 1e and $>$ Fig. 1f are illustrative of a PNS case with long-term follow-up.

\section{Discussion}

The healing process of the post-ESD defect is still not well understood. The majority of patients that undergo curative enbloc endoscopic resection of superficial gastric neoplasms develop homogeneous and flat epithelized scar covered by a regular-appearing mucosa with correspondent fibrosis. However anecdotally we noted, in two different institutions, that certain patients would develop aberrant and protuberant polypoid nodules in the post-ESD scar. Biopsy of these nodules demonstrated regenerative and hyperplastic tissue growth, and we named this entity polypoid nodule scar (PNS) $[3,4]$. Presence of PNS may have direct clinical implications because it may render evaluation of post-ESD neoplastic recurrence difficult. To better define the incidence and clinical significance of PNS we conducted this large multicenter study including five high-volume ESD expert centers ( 4 in Asia and 1 in Brazil). Our main findings are that PNS occurred after curative gastric ESD in close to $1.2 \%$ of the cases. Importantly, PNS occurred only after ESD performed for antral lesions. If we consider only ESD procedures carried out for tumors located in the antrum, the proportion of PNS cases was 3.1\%. Importantly, in no patient with PNS neoplastic changes were detected and the lesions remain stable on surveillance endoscopy but in approximately $18 \%$, PNS disappeared with time. 
In this study we noted a wide variation in tumor size and ESD defect leading to development of PNS, and likewise the size of PNS also presented some variation. The smallest tumor treated by ESD that developed PNS measured only $8 \mathrm{~mm}$, and the largest measured $55 \mathrm{~mm}$. Apparently tumor size or post-ESD ulcer size are not important factors in development of PNS.

In our initial observation we did not detect a clear association between $H$. pylori status and development of PNS, since $57 \%$ of the patients with PNS tested positive for $\mathrm{H}$. pylori. Now our current much larger investigation reconfirms that it is unlikely that $H$. pylori status influences occurrence of PNS, since half of our patients were $H$. pylori-positive. The effect of $H$. pylori eradication therapy on PNS remains to be elucidated in future studies.

The precise mechanism that explains why some individuals develop PNS and others do not is still poorly understood. Similar aberrant PNS also have been noted to occur after healing of benign gastric ulcers located in the antrum in up to $6 \%$ of cases [8], leading to unnecessary gastric resection in the past [9]. Other investigators have observed development of protruded granulation tissue in healing ulcer after medical treatment either with histamine-2 receptor antagonist (H2RA) [10] or PPI $[11,12]$. Interestingly, the protruded granulation tissue after benign ulcer healing may disappear or remain unchanged for a long time $[11,13]$ Histological findings of the polypoid nodule at benign ulcer scar similarly are indicative of hyperplastic regenerative mucosa on fibrotic tissue [9].

Of note in this study, the proportion of PNS cases varied from $0.15 \%$ to $11.4 \%$ among the participating institutions, although in three Japanese centers the appearance was around $2 \%$. The reason for this wide variation may be related to differences in ESD technique, total number of cases and operator experience, postoperative care or even ethnic difference. To assess the influence of a learning curve effect on the appearance of PNS, we divided the study period into two phases (20032010 and $2011-2016)$. Only in six patients (21.4\%) ESD was undertaken in the early period (2003 to 2010). The great majority of cases occurred after 2011 , when all operators had already overcome the learning curve for gastric ESD (first 40 cases). It is important to note that the total number of PNS cases included in this study is still small and most likely insufficient to allow a reliable statistical analysis. Future studies with larger numbers of patients will be needed to address possible predisposing factors to PNS appearance, such as ESD technique, type of knife or operator's experience.

The great majority of patients undergoing gastric ESD are routinely treated postoperatively with PPI to accelerate the healing process, a clinical management adopted universally and in the five centers involved in this study [14]. However, there was some variation among the postoperative medical management, with Center 1 administering sucralfate for 2 weeks and Center 3 adding sucralfate and sodium alginate for 1 week and rebapimide for 8 weeks. PPI accelerates ulcer healing mainly due to potent gastric acid secretion inhibition. However, PPI also increases cyclooxigenase-2 (COX-2) expression and prostaglandin E synthases in the ulcerated mucosa [15]. COX-2-generated prostaglandin E2 stimulates expression of growth factors such as vascular endothelial growth factor (VEGF) [16], hepatocyte growth factor (HGF) [17], and basic fibroblast growth factor (bFGF) [18] in the mucosa. This accelerated mucosal repair and angiogenesis may contribute to nodular overgrowth of the regenerative mucosa. The influence of other medications, such as sucralfate, sodium alginate or rebapimide on the scarring process of ESD defects, need to be addressed in future studies.

A unique universal characteristic of PNS is that this aberrant scar only occurs after ESD performed in the distal stomach (antrum or incisure). No PNS was observed after resection of lesions located in the gastric body, fundus or cardia. Likewise, so far, we have not observed this finding after esophageal or colorectal ESD. The reason for this phenomenon is unclear. We postulate that gastric peristalsis and/or bile reflux may enhance development of PNS in the antrum. Moreover, the submucosal layer in the antrum is thicker; therefore, inflammatory or regenerative reaction in the submucosa may be more obvious in the antrum than in the corpus of fundus.

Our study was not without limitations. It was a retrospective analysis of prospectively collected data. Although the endoscopic database of each center participating in this study was organized and continuously updated, some cases of PNS may have been missed during data extraction. Furthermore, only patients that returned for follow-up were enrolled, therefore other potential cases of PNS may have been missed. Finally, there might be overlooked cases of PNS that were not characterized by the expert endoscopist, or even referred to surgery or endoscopic intervention, due to fear of recurrence or malignancy, particularly in the early period of the study when ESD was not yet a well-established procedure. These limitations suggest that the precise incidence rate for PNS may be even greater than the rate of $1.2 \%$ observed in this multicenter study, and a prospective study is needed to further investigate the incidence rate of PNS and its relationship with postoperative medical management, $H$. pylori status/eradication therapy and patient/tumor characteristics.

\section{Conclusion}

In conclusion, this large multicenter study demonstrates that after curative ESD, PNS develops only after removal of lesions in the distal stomach in about $3.1 \%$ of cases. Endoscopists performing ESD should be aware that PNS may develop and although it can have an alarming appearance, it is a benign condition. Based on our data, patients with PNS can be reassured and followed with annual endoscopic surveillance to search for metachronous lesions and to examine and biopsy the ESD scar.

\section{Competing interests}

None 


\section{References}

[1] Pimentel-Nunes P, Dinis-Ribeiro M, Ponchon T. Endoscopic submucosal dissection: European Society of Gastrointestinal Endoscopy Guideline. Endoscopy 2015; 47: 829-548

[2] Ono H, Yao K, Fujishiro M et al. Guidelines for endoscopic submucosal dissection and endoscopic mucosal resection for early gastric cancer. Dig Endosc 2016; 28: 3-15

[3] Arantes V, Uedo N, Pedrosa MS. Polypoid nodular scar after endoscopic submucosal dissection in the gastric antrum. Rev Gastroenterol Mex 2017; 82: 267 - 269

[4] Arantes V, Uedo N, Pedrosa MS et al. Clinical relevance of aberrant polypoid nodule scar after endoscopic submucosal dissection. World ] Gastrointest Endosc 09 2016; 16: 628-634

[5] Piñeros EAF, Arantes V, Toyonaga T. Endoscopic submucosal dissection of early gastric cancer: State of the art. Rev Col Gastroenterol 2012; 27: $194-214$

[6] Japanese Gastric Cancer Association. Japanese classification of gastric carcinoma: 3rd. English edition. Gastric Cancer 2011; 14: $101-112$

[7] Yamamoto H. Endoscopic submucosal dissection - current success and future directions. Nat Rev Gastroenterol Hepatol 2012; 9: 519 529

[8] Ito S, Kishi S, Ishikawa K et al. A case of gastric ulcer scar with type Ilalike elevation in center of the lesion. Gastroenterol. Endosc 1974; 16: $194-197$

[9] Ito S, Ishikawa I, Kishi S et al. Elevated type gastric ulcer scar. Gastroenterol. Endosc 1976; 18: $420-427$

[10] Kato H, Hattori K, Nishikawa $\mathrm{H}$ et al. Endoscopic study on healing of gastric ulcer by the treatment with $\mathrm{H} 2$-blocker. Gastroenterol Endosc 1986; 28: $2291-2296$
[11] Nakamura T, Tsukamoto Y, Yamanaka T et al. A study on the whitish protrusion appearing in the base of peptic ulcer during the administration of proton pump inhibitor. Gastroenterol Endosc 1992; 34: $1548-1554$

[12] Ashida K, Osaka N, Tei H et al. Studies on the mechanism of the protrusion of the base of the ulcer during the administration of omeprazole. Gastroenterol Endosc 1989; 31: 1776-1782

[13] Tanaka T, Kimura M, Akiyama T et al. Long follow-up study of elevated scar of acute antral kissing ulcers. Gastroenterol Endosc 1984; 26: $1534-1537$

[14] Uedo N, Takeuchi Y, Yamada T et al. Effect of a proton pump inhibitor or an $\mathrm{H} 2$-receptor antagonist on prevention of bleeding from ulcer after endoscopic submucosal dissection of early gastric cancer: A prospective randomized controlled trial. Am J Gastroenterol 2007; 102: $1610-1616$

[15] Okazaki M, Shimizu I, Ishikawa M et al. Gastric mucosal levels of prostaglandins and leukotrienes in patients with gastric ulcer after treatment with rabeprazole in comparison to treatment with ranitidine. J Med Invest 02 2007; 54: 83-90

[16] Wallace JL, Devchand PR. Emerging roles for cyclooxygenase-2 in the gastrointestinal mucosal defense. Br J Pharmacol 2005; 145: 275 282

[17] Brzozowski T, Konturek PC, Konturek SJ et al. Involvement of cyclooxygenase (COX)-2 products in acceleration of ulcer healing by gastrin and hepatocyte growth factor. J Physiol Pharmacol 2000; 51: 751 773

[18] Sakai Y, Fujita K, Sakai H et al. Prostaglandin E2 regulates the expression of basic fibroblast growth factor messenger RNA in normal human fibroblasts. Kobe J Med Sci 2001; 47: 35-45 